\title{
Derecho y activación: dos claves de evolución
}

\author{
Law and activation: two keys of development \\ Gemma Zabaleta Areta \\ Consejera de Empleo y Asuntos Sociales del Gobierno Vasco \\ e-azurrmendi@ej-gv.es
}

Recibido: 29/09/2011

Revisado: $15 / 11 / 2011$

Aceptado: 28/11/2011

Disponible on line: 15/02/2012

\begin{abstract}
Resumen
La comunidad autónoma del País Vasco se encuentra inmersa en un activo proceso de desarrollo normativo, planificación de servicios y cambio organizativo, orientado hacia dos objetivos principales, cuya progresiva consecución supondrá un avance fundamental en la consolidación del sistema vasco de Servicios Sociales y del sistema vasco de Garantía de ingresos e inclusión social. El primero de estos objetivos es garantizar el ejercicio efectivo del derecho subjetivo a los Servicios Sociales, proclamado por la Ley 12/2008, de 5 de diciembre, de Servicios Sociales, lo que sitúa en el eje central del desarrollo normativo la regulación de la Cartera de prestaciones económicas y de servicios y, en el núcleo de la planificación estratégica, el mapa de Servicios Sociales. El segundo objetivo básico es reforzar la centralidad del empleo contemplada en la Ley 18/2008, de 23 de diciembre, para la Garantía de ingresos y para la inclusión social, lo que ha se ha materializado, en el marco de una reforma legal, en el traslado al ámbito del empleo, de los componentes esenciales del sistema, a saber: la gestión y tramitación la renta de garantía de ingresos y de la prestación complementaria de vivienda, así como de las funciones asociadas a la suscripción y seguimiento de los convenios de inclusión activa; este cambio organizativo pretende dotar al sistema de instrumentos mejor adaptados a su objetivo final de inclusión, y refuerza y visibiliza un cambio de estrategia que supone un acercamiento del modelo vasco a los planteamientos imperantes en Europa.
\end{abstract}

Palabras clave: derecho a los servicios y prestaciones sociales, catálogo y cartera de prestaciones y servicios, planificación y despliegue territorial, gestión de prestaciones económicas de garantía de ingresos,m edidas de activación.

\begin{abstract}
The Basque Autonomous Community is undertaking an active process of policy development, service planning and organizational change with two main objectives that will progressively lead to the consolidation of the Basque Social Service System and the Basque System of Minimum Income Guarantees and Social Inclusion. The first of these two objectives is to guarantee that the right to social services, as proclaimed by the Social Services Act (2008), is made effective. This makes control of the Social Service Description List the central axis of policy development, and the Social Services Map the core of strategic planning. The second basic objective of the process undertaken is to put employment at the center of social inclusion, as established in the Minimum Income Guarantee and Social Inclusion Act (2008). In order to achieve this goal, legal reform has been undertaken which has transferred the essential components of the System, those being the management and processing of the Minimum Income Support and its complementary housing benefit, as well as the signing and monitoring of contracts for active inclusion, to the area of employment. This organizational change intends to provide the System with better instruments to achieve inclusion, which is the System's final objective. It also makes visible and reinforces a change in strategy that brings the Basque model closer to prevailing European approaches.
\end{abstract}

Keywords: Right to social services and social benefits, Services and Benefits Description List, Planning and mapping of services, Management and administration of minimum income benefits, Activation measures.

Referencia normalizada: Zabaleta Areta, G. (2012): «Derecho y activación: dos claves de evolución». Cuadernos de Trabajo Social, 25(1): 87-101.

Sumario: Presentación. 1. La articulación del sistema vasco de Servicios Sociales. Definición y la delimitación del derecho: el catálogo y la cartera de prestaciones y servicios. 2. Un giro en el sistema vasco de Garantía de ingresos e inclusión social: la activación como clave de la inclusión. 3. Conclusiones. 4. Referencias bibliográficas. 


\section{Presentación}

La comunidad autónoma del País Vasco se ha convertido, en los dos últimos años, en el escenario de innovaciones de gran calado, que marcan una nueva etapa en el desarrollo de los dos pilares básicos de las políticas públicas en el ámbito de la acción social:

- en el caso del sistema vasco de Servicios Sociales, las innovaciones vienen de la mano del reconocimiento del derecho subjetivo, que introdujo la Ley 12/2008, de 5 de diciembre, de Servicios Sociales, y se vehiculan desde los instrumentos de desarrollo normativo orientados al despliegue del sistema hasta alcanzar su universalización;

- en el caso del sistema vasco de Garantía de ingresos e inclusión social, el avance se centra en el reforzamiento de la asociación establecida entre las prestaciones económicas de garantía de ingresos y la activación, en particular aquella orientada al empleo, en el marco de las recién transferidas competencias en el ámbito de las políticas activas de empleo.

En ambos frentes, el reto es de envergadura y responde a la necesidad de garantizar, a la vez, la progresión de los sistemas y su sostenibilidad, lo que necesariamente exige su percepción y su aceptación como instrumentos necesarios y garantistas de protección pero también de justicia social. Trataré aquí de dar las claves de estos procesos que se están liderando desde el Departamento de Empleo y Asuntos Sociales.

\section{La articulación del sistema vasco de Ser- vicios Sociales.}

La Ley de Servicios Sociales, aprobada a finales de la legislatura anterior - con un apoyo muy activo por parte del grupo socialistaobedeció al deseo de aportar elementos de avance hacia un modelo de derecho subjetivo y hacia un sistema estructurado de Servicios Sociales, con nuevas líneas de actuación y ámbitos de innovación, capaces de responder a nuevos retos y demandas sociales. El texto legal -muy denso y exhaustivo para evitar las ambigüedades e indefiniciones que tanto se habían reprochado a los textos previos - articula una estructura compleja (cuadro 1).

La complejidad de esta estructura determina también, lógicamente, una gran compleji- dad tanto en la propia creación o adaptación de los elementos que la integran como en el funcionamiento coordinado y coherente del conjunto. Aunque todos sus componentes resultan esenciales, en tanto que complementarios los unos de los otros, no cabe duda que el núcleo del modelo se sitúa en los elementos de garantía y en ellos me centro aquí.

El reconocimiento del derecho subjetivo a los Servicios Sociales constituye el eje de todo el entramado: es el que determina el paso de un modelo en el que el acceso a la mayoría de los servicios está sujeto a disponibilidad presupuestaria (y, en última instancia, a la discrecionalidad administrativa), a otro en el que se reconoce el derecho de acceso y se impone a las administraciones competentes la obligación de proveer los servicios. Es también el que genera importantes consecuencias, no sólo en términos de ampliación de la cobertura e intensidad de la red de prestaciones y servicios hasta alcanzar la universalización, sino también en términos de capacidad de gestión de un volumen de demandas que no podrá si no evolucionar en sentido creciente.

Para la construcción, la puesta en marcha y el funcionamiento de este nuevo modelo, se adopta un marco filosófico con dos principales vectores de referencia: el enfoque comunitario de la atención y los principios rectores del sistema (cuadro 2).

Con este encuadre - esencial para la correcta comprensión del modelo- el reconocimiento del derecho alcanza su concreción a través de una serie de instrumentos dirigidos, los unos, a la definición y delimitación del derecho, y los otros, orientados a la planificación y el despliegue del sistema vasco de Servicios Sociales para garantizar el ejercicio de ese derecho, en condiciones de igualdad, en el conjunto del territorio autonómico. La determinación de su alcance ha constituido, a lo largo de los dos últimos años, una de las prioridades máximas del Departamento de Empleo y Asuntos Sociales en sus esfuerzos de diseño del sistema en el marco de un intenso proceso de diseño, colaboración y negociación interinstitucional, clave esta última ineludible en un marco como el de los servicios sociales vascos, caracterizados por la convergencia competencial de los tres niveles administrativos: el autonómico, el foral y el municipal. 


\begin{tabular}{|c|c|c|}
\hline \multirow{5}{*}{$\begin{array}{l}\text { Elementos } \\
\text { de garantía }\end{array}$} & Derecho Subjetivo & $\begin{array}{l}\text { - Tutela judicial } \\
\text { — Universalización } \\
\text { de servicios }\end{array}$ \\
\hline & Sistema Vasco de Servicios Sociales & \\
\hline & Enfoque comunitario & — Proximidad de la atención \\
\hline & Catálogo de Prestaciones y Servicios & $\begin{array}{l}\text { - Cartera de Prestaciones } \\
\text { y Servicios }\end{array}$ \\
\hline & Planificación de servicios & $\begin{array}{l}\text { - Plan Estratégico } \\
\text { - Mapa de Servicios }\end{array}$ \\
\hline
\end{tabular}

\begin{tabular}{|c|c|c|}
\hline \multirow{4}{*}{$\begin{array}{l}\text { Elementos } \\
\text { de organización }\end{array}$} & \multicolumn{2}{|l|}{ Distribución competencial } \\
\hline & Coordinación & $\begin{array}{l}\text { - Órgano Interinstitucional } \\
\text { de Servicios Sociales }\end{array}$ \\
\hline & Participación & $\begin{array}{l}\text { - Consejo Vasco de Servi- } \\
\text { cios Sociales }\end{array}$ \\
\hline & Colaboración con la iniciativa privada & $\begin{array}{l}\text { - Régimen de concierto } \\
\text { - Convenios } \\
\text { - Contratos } \\
\text { - Acuerdos marco }\end{array}$ \\
\hline
\end{tabular}

Elementos de mejora

Elementos de control

\section{Elementos de financiación \\ de control}

- Observatorio Vasco de Servicios Sociales

- Sistema Vasco de Información en Servicios Sociales

- Formación

- Calidad

$\{$ - Alta inspección

- Autorización, registro, homologación e inspección de servicios

- Régimen de infracciones y sanciones

Cuadro 1. Ley 12/2008, de 5 de diciembre, de servicios sociales de la CAPV. Elementos básicos. Fuente: La Situación de los Servicios Sociales de Base en la CAPV. Informe extraordinario del Ararteko, VitoriaGasteiz, 2010. 
- La adaptación de los recursos y las intervenciones a las características de cada comunidad local.

- La atención de las personas en su entorno habitual, preferentemente en el domicilio, y la articulación, cuando la permanencia en éste no resulte viable, de alternativas residenciales idóneas, por su tamaño y proximidad, a la integración en el entorno.

- El diseño del tipo de intervención adecuada a cada caso, sobre la base de una evaluación de necesidades y en el marco de un plan de atención personalizada.

- La asignación a cada persona o familia, de un profesional o una profesional de referencia en el sistema vasco de Servicios Sociales, al objeto de garantizar la coherencia de los itinerarios de atención y la coordinación de las intervenciones.

- El carácter interdisciplinar de la intervención.

- La incorporación, en todas las prestaciones, servicios, programas y actividades, del enfoque preventivo.
- Principio de responsabilidad pública, en virtud del cual los poderes públicos garantizarán la disponibilidad y el acceso a las prestaciones y servicios previstos en el catálogo.

- Principio de universalidad, en virtud del cual los poderes públicos garantizan el derecho a una serie de prestaciones y servicios recogidos en el catálogo a todas las personas definidas como titulares, siempre que cumplan los requisitos específicos que se establezcan a nivel reglamentario para cada uno de ellos.

- Principio de igualdad, orientado a asegurar, atendiendo a criterios de justicia territorial, como mínimo la cobertura de prestaciones y servicios que defina el mapa de Servicios Sociales al objeto de asegurar una distribución homogénea de los recursos en el territorio autonómico.

- Principio de proximidad, definitorio del enfoque comunitario de la atención, según el cual, la prestación de los servicios sociales, cuando su naturaleza lo permita, responderá a criterios de máxima descentralización y desconcentración.

- Principio de atención personalizada e integral y de continuidad de la atención, como elemento constitutivo tanto del modelo de atención comunitaria como del procedimiento básico de intervención.

- Principio de interdisciplinariedad, que, con el fin de garantizar el carácter integral de la atención prestada y la aplicación racional y eficiente de los recursos públicos, promueve el trabajo en equipo y la integración de las aportaciones de las diversas profesiones.

Cuadro 1. Vectores del modelo. Elaboración propia.

\subsection{Definición y delimitación del derecho: el catálogo y la cartera de prestaciones y servicios}

Un sistema que proclama el derecho de las personas a los Servicios Sociales debe necesariamente situar su piedra angular, en la definición y en la delimitación de ese derecho, es decir, en determinar:

- qué tipos de servicios y prestaciones, de todos los existentes en el ámbito de los Servicios Sociales, quedan incluidos en el mismo y recaen, por lo tanto, en la responsabilidad pública;
- qué personas son las destinatarias potenciales de cada uno de esos tipos de prestaciones y servicios;

- qué requisitos deben reunir esas personas para poder acceder a cada uno de ellos.

Estos elementos básicos constituyen el objeto del catálogo y de la cartera de prestaciones y servicios.

El catálogo establecido en la propia Ley de Servicios Sociales ${ }^{1}$ se limita a listar los servicios y prestaciones económicas que recaen en la responsabilidad pública, imponiendo a las administraciones públicas competentes - Go-

\footnotetext{
${ }^{1}$ Véase Anexo I.
} 
bierno vasco, diputaciones forales o ayuntamientos, según los casos - el deber de garantizar la provisión de dichos servicios y prestaciones económicas, es decir, su existencia y disponibilidad, sin perjuicio de que para su prestación puedan recurrir a la colaboración de la iniciativa privada, con y sin ánimo de lucro, salvo en el caso de las prestaciones de acceso y primera acogida de las demandas y de las directamente asociadas a la coordinación de caso que serán siempre de gestión pública directa. Sólo lista las categorías o tipos de servicios y prestaciones económicas: ni los define, ni distingue modalidades, ni especifica la población destinataria en cada caso, entendiendo que la misma deberá cumplir las condiciones generales de titularidad previstas en el texto legal.

La amplitud de esa formulación legal obedecía, lógicamente, a la necesidad de evitar disposiciones en exceso ligadas a la realidad actual para dejar espacio a la evolución del sistema y a su progresiva adaptación y ajuste a las necesidades de la población y para dar cabida a las nuevas fórmulas de atención que pudieran ir perfilándose con el tiempo, sin que dicha evolución exigiera sucesivas reformas legales. De ahí que la ley previera un instrumento de desarrollo del catálogo - la cartera de prestaciones y servicios - , más fácilmente ajustable y modificable al hilo de dicha evolución.

De ahí que, a lo largo de estos dos últimos años y en paralelo con otros avances normativos imprescindibles ${ }^{2}$, la labor del Departamento de Empleo y Asuntos Sociales se haya centrado en alcanzar, en el marco de un intenso proceso de debate y aportaciones, una regulación de la cartera que fuera compartida por el conjunto de las administraciones públicas competentes, y que, a su vez, pudiera enriquecerse con aportaciones realizadas desde el Tercer Sector de acción social. Es evidente que la convergencia de los tres niveles administrativos - autonómico, foral y municipal- en la distribución competencial vigente en el ámbito de los Servicios Sociales añade dificultad a la construcción del sistema: la distribución com- petencial para la provisión de servicios y prestaciones del catálogo es, en realidad, un sistema de vasos comunicantes, en cuyo marco todo aquello que no se incluye en la definición de un determinado servicio y, por lo tanto, en la competencia de un determinado nivel institucional, recae, necesariamente, en la competencia de otro nivel, y en ese contexto, alcanzar consensos resulta una tarea ardua, máxime en un ciclo de crisis económica como el que vivimos. En la actualidad, y al cabo de un largo proceso de debate, el Gobierno vasco cuenta con un primer texto, que, esta vez, se someterá ya a un proceso formal de aportaciones, con el objetivo de iniciar la tramitación del decreto en un plazo breve.

Sin entrar aquí en los detalles de la cartera, y mucho menos de los diferentes servicios y prestaciones, creo importante destacar algunos elementos de especial relevancia para su comprensión y para su aplicación por cuanto que modulan fuertemente la orientación que se pretende dar a este instrumento:

- El primero se refiere a la estructuración de las situaciones atendidas. Todos los servicios y prestaciones económicas van referidas a uno o varios de los cuatro grandes ámbitos de atención previstos en la ley de Servicios Sociales: la dependencia, la protección, la exclusión y la emergencia social, diferenciándose en los tres primeros entre las situaciones de riesgo y las situaciones ya declaradas de necesidad, con el fin de facilitar la delimitación de los derechos en aras de la seguridad jurídica. Tal diferenciación lleva aparejada, lógicamente, la necesidad de diseñar una batería de instrumentos comunes, indicadores y criterios técnicos aplicables por el conjunto de las administraciones públicas vascas.

- El segundo elemento tiene que ver con el valor que se otorga a la prescripción técnica de la persona profesional de referencia. Efectivamente, el texto condiciona el acceso no sólo al cumplimiento de los requisitos administrativos y de necesidad que correspondan, en fun-

\footnotetext{
${ }^{2}$ Desde el inicio de la legislatura se han dado importantes pasos para la consolidación del entramado institucional previsto en la ley: se han aprobado los decretos reguladores del órgano interinstitucional de Servicios Sociales y del Consejo vasco de atención sociosanitaria, y se ha avanzado muy considerablemente en la regulación, todavía en curso, de la participación económica de las personas usuarias y del régimen de concierto.
} 
ción del tipo de servicio o prestación, sino también a su idoneidad para responder, mejor que otros, a la situación de necesidad. En otros términos, el reconocimiento de la existencia de una situación de riesgo o de una situación reconocida de dependencia, desprotección o exclusión, determinará la gama de servicios y prestaciones económicas del sistema vasco de Servicios Sociales, susceptibles de responder a dicha situación, pero la determinación del servicio y prestación o de los servicios y prestaciones económicas específicas a los que se acceda dependerá de su grado de idoneidad para dar respuesta a las necesidades detectadas. Dicho grado de idoneidad dependerá, a su vez, de dos elementos: la mayor o menor adecuación del tipo de servicio o prestación a la situación personal, familiar, convivencia y relacional y, en su caso, a las características de la vivienda, y la mayor o menor capacidad del servicio o prestación para ofrecer el nivel de intensidad de apoyo requerido.

Pues bien, la idoneidad del servicio o de la prestación económica que constituye, por así decirlo, el filtro final de acceso deberá quedar recogida en la prescripción técnica de la o del profesional de referencia, lo cual refleja la relevancia del papel que se le atribuye en el acceso al sistema, aun cuando se prevea que, para su determinación, deberá contar con la participación de la persona o la familia afectada y tener en cuenta, en lo posible, la preferencia que manifiesten.

- Un tercer elemento fundamental viene dado por el carácter prioritario que, en el marco de la determinación de la idoneidad del servicio o de la prestación, se otorga a la atención en el marco comunitario. Así, para garantizar la aplicación efectiva de esta prioridad se prevé que, en los casos en los que la persona profesional de referencia opte, en su prescripción técnica, por una solución residencial, ya se trate de servicios de alojamiento de atención primaria o de centros residenciales de atención secundaria, deberá justificar la no adecuación de una fórmula de atención más susceptible de garantizar la permanencia de la persona usuaria en su entorno habitual.
- Un cuarto elemento reseñable es el protagonismo que adquiere la personalización de la atención, materializada, más allá de los principios, en la asignación de una persona profesional de referencia y en la elaboración de un plan de atención personalizada. Sin ser una novedad ni en el modelo vasco ni en su cuerpo normativo $^{3}$, su inclusión en una norma como el Decreto de cartera que, por su centralidad, se erige en rectora del sistema, le dota de un significado y de una importancia hasta ahora ausentes, que debería impedir que tales pautas de funcionamiento tiendan a considerarse como simples recomendaciones, propias, como tales, del ámbito de lo deseable y de lo discrecional.

- A nivel procedimental, la norma incluye también una especificidad digna de mención. Prevé, además del procedimiento ordinario y del procedimiento aplicable en situaciones de urgencia social, una tercera modalidad, intermedia, denominada «procedimiento de acceso urgente» que pretende dar respuesta a situaciones que, sin constituir urgencias sociales, requieren una atención prioritaria y actuar con mayor celeridad de la prevista en el procedimiento ordinario, dejando para un segundo momento la realización del conjunto de las actuaciones de valoración y verificación de requisitos. Esperamos que esta vía permita dar solución a algunas dificultades observadas en la actualidad.

Por último, se plantea en relación con la cartera, la cuestión fundamental de su efectividad, en un marco legal en el que la universalización del sistema no se prevé hasta el año 2016. Pues bien, la previsión es que, una vez aprobado, el decreto iniciará su aplicación, pero ésta será progresiva, ya que la efectividad plena del derecho a los servicios y prestaciones del sistema sólo podrá ejercitarse a partir de la fecha en que se alcance la universalización del mismo. Esto significa que es necesario prever cómo se ejercitará el derecho a los servicios y prestaciones entre ambos momentos y, para ello, el texto remite la definición de esa gradualidad al órgano interinstitucional de Servicios Sociales, con el fin de implementar una

\footnotetext{
${ }^{3}$ Se incluían en el Decreto 64/2004, de 6 de abril, de Carta de Derechos y Obligaciones de las Personas Usuarias y Profesionales de los Servicios Sociales en la Comunidad Autónoma del País Vasco y el Régimen de Sugerencias y Quejas (BOPV de 23 de abril de 2004).
} 
fórmula de avance que garantice el principio de igualdad en el acceso en el conjunto del territorio autonómico.

Durante ese periodo transitorio, las administraciones públicas vascas reconocerán, si se cumplen los requisitos correspondientes, el derecho de acceso de la persona demandante a los servicios y/o prestaciones económicas, si bien el derecho reconocido sólo se hará efectivo a partir de la fecha que corresponda en cada caso. Lógicamente, lo anterior no será de aplicación a los servicios y prestaciones económicas previstas en el marco del Sistema para la Autonomía y Atención a la Dependencia, salvo cuando dichas previsiones resulten más ventajosas para la persona solicitante que el calendario de acceso recogido en la disposición final primera de la Ley 39/2006, de 14 de diciembre, de promoción de la Autonomía personal y Atención a las personas en situación de Dependencia.

\subsection{La planificación y el despliegue territo- rial: el Plan estratégico y el mapa de Servi- cios Sociales}

Simultáneamente al proceso de la cartera - unas veces en paralelo y otras conjuntamente con él- hemos avanzado en la definición de los ejes estratégicos para el desarrollo del sistema y en el diseño del despliegue territorial de la red; estos contenidos han dado cuerpo al Plan estratégico de Servicios Sociales y, dentro de éste, al mapa de Servicios Sociales.

El Plan estratégico propiamente dicho ya se encuentra consensuado entre los tres niveles administrativos. Este instrumento, esencial en la arquitectura del sistema por cuanto que marca el rumbo de avance, se articula en: 7 ejes básicos de intervención, que definen las áreas prioritarias de actuación; 29 objetivos estratégicos, que concretan los avances que han de darse en cada uno de esos ejes; 80 acciones, orientadas a alcanzar los objetivos estratégicos, especificándose para cada una de ellas la entidad responsable, los aspectos prioritarios, los indicadores de evaluación y, en determinados casos, la cuantificación del coste previsto para su realización ${ }^{4}$.

El Plan esboza, además, una línea de acción básica o transversal que - por acuerdo de to- das las instituciones que ha intervenido en su definición - ha querido distinguirse por su carácter estratégico del conjunto de las medidas que conforman el Plan: es una línea de acción relativa a la financiación y la sostenibilidad económica del sistema vasco de Servicios Sociales, que, por su carácter esencial, todas las instituciones consideran previa al resto de las acciones. A este respecto, me parece indispensable apuntar que, inevitablemente, el Plan se desarrolla en un marco de crisis económica y reducción de la capacidad de gasto público que, por una parte, dificultan hacer frente a los retos planteados y, por otra, generan un incremento en la demanda de determinadas prestaciones y servicios, haciendo más necesaria, si cabe, la adopción de medidas que permitan mejorar la eficiencia en el uso de los recursos. Con todo, consideramos indispensable insistir en la idea de que el desarrollo de los Servicios Sociales debe considerarse en términos de oportunidad y de inversión, dado el potencial de generación de empleo de los Servicios Sociales, sus elevados niveles de retorno económico y su capacidad para la prevención de problemáticas que implican un coste personal, social y económico muy elevado.

La dimensión estratégica aportada por el Plan se liga, necesariamente, a un instrumento capaz de hacer visible, en el territorio autonómico, el alcance de las medidas: ese ha sido el cometido del mapa de Servicios Sociales, específicamente centrado en el despliegue territorial y en el dimensionamiento del sistema, determinantes a su vez de las previsiones de coste que le quedan asociadas y que se recogen en la Memoria económica.

Concretamente, el mapa establece las principales magnitudes de los servicios que conforman el catálogo de Servicios Sociales en relación con cinco elementos básicos:

- El ámbito territorial, es decir, el nivel de la estructura territorial del sistema de Servicios Sociales en el que, con objeto de garantizar la homogeneidad en el acceso a los servicios y prestaciones, se deben concretar los índices de cobertura y de densidad establecidos para cada servicio o prestación. La ordenación territorial sobre la que se articula el mapa de Servicios

\footnotetext{
${ }^{4}$ Todos ellos se detallan en el Anexo II.
} 
Sociales se basa en la asignación a cada uno de los servicios del catálogo de servicios y prestaciones de un grado determinado de proximidad:

- Servicios y prestaciones centralizados.

- Servicios y prestaciones de baja proximidad.

- Servicios y prestaciones de proximidad media.

- Servicios y prestaciones de alta proximidad.

- Servicios y prestaciones de máxima proximidad.

Cada uno de los niveles de proximidad establecidos se vincula, a su vez, a un nivel territorial específico. Implica que la cobertura, intensidad o densidad que se determina en el mapa con carácter de mínimos para cada uno de los servicios del catálogo ha de entenderse referida a ese ámbito territorial concreto. Se incluye una tabla que establece el grado de proximidad de los diferentes servicios y prestaciones del sistema ${ }^{5}$.

- La cobertura o, en el caso de los servicios dirigidos al conjunto de la población, la dotación de profesionales que se precisa para garantizar, en cada uno de los ámbitos territoriales citados, la universalidad de los servicios.

- La intensidad, en aquellos servicios como la ayuda a domicilio- en los que constituye un elemento definitorio e indispensable para garantizar la adecuada cobertura de las necesidades.

- La ratio de atención, en número de profesionales por plaza o por persona usuaria, como medio para procurar una atención de calidad.

- La densidad de centros - en aquellos servicios en los que resulte pertinente- como criterio rector del despliegue territorial en el área geográfica de implantación y como criterio delimitador del tamaño de los mismos.

A la hora de establecer los niveles mínimos de cobertura, ratios, intensidad o densidad para cada tipo de servicio incluido en el mapa, se ha recurrido a la literatura especializada y a los criterios profesionales de buena práctica. En el caso específico de las coberturas, los valores mínimos se han determinado a partir del principio de que, una vez culminado en 2016 el periodo de universalización, la red de Servicios
Sociales de responsabilidad pública deberá ser capaz de atender la práctica totalidad de la demanda potencial prevista para ese año. De esta forma, la determinación de los recursos que será necesario habilitar para ese año se deriva de un estudio prospectivo de la demanda potencial de servicios - a partir de la aplicación de una serie de hipótesis de evolución a la actual prevalencia de las contingencias que cubre el sistema- y de la distribución del conjunto de esa demanda potencial en una serie de paquetes de atención, integrados por uno o más servicios o prestaciones, de acuerdo a la gravedad de las situaciones y a los principios generales del sistema (proximidad, atención comunitaria, énfasis en los servicios domiciliarios y de atención primaria, prioridad de los servicios de atención directa frente a la percepción de prestaciones económicas, etc.).

Estos son los parámetros básicos del mapa en su versión actual. Con todo, al igual que la cartera, esta propuesta se encuentra todavía pendiente de un proceso de aportaciones, que completará y cerrará el debate que se ha desarrollado a lo largo de los dos últimos años, dando paso a su tramitación. En el marco de ese proceso, no es imposible que, atendiendo a las posibilidades reales del Sistema, algunos de estos parámetros pierdan protagonismo y adquieran un valor más referencial, de objetivo deseable, en lugar de constituirse en elementos de aplicación obligatoria.

\section{Un giro en el sistema vasco de Garantía de ingresos e inclusión social: la activación como clave de la inclusión}

Junto a la ingente labor de articulación del sistema vasco de Servicios Sociales en torno al derecho subjetivo, el Departamento de Empleo y Asuntos Sociales está haciendo frente a otro gran reto desde el comienzo de la legislatura: el del afianzamiento del sistema vasco de Garantía de ingresos e inclusión social. Presenta una doble vertiente: por un lado, la regulación del nuevo marco prestacional contenido en la Ley 18/2008, de 23 de diciembre, para la Garantía de ingresos y para la inclusión social; por otro, el reforzamiento de la asociación entre las prestaciones económicas y la activación de las personas beneficiarias.

\footnotetext{
${ }^{5}$ Véase en el Anexo III.
} 
En cuanto a lo primero, los decretos reguladores ya entraron en vigor a lo largo del 2010 y comienzo de $2011^{6}$, iniciándose así la andadura de la nueva estructura prestacional establecida en la ley. Esta nueva fórmula diferencia dos grupos de prestaciones: las prestaciones económicas de derecho, que incluyen la renta de garantía de ingresos y la prestación complementaria de vivienda, y las ayudas económicas subvencionales, es decir, las ayudas de emergencia social; a su vez, la renta de garantía de ingresos se subdivide en dos modalidades diferenciadas en función de la existencia o no de ingresos en la unidad de convivencia y, en su caso, en función de la procedencia de dichos ingresos: la renta básica para la inclusión y protección social y la renta complementaria de ingresos de trabajo.

Estos cambios con respecto a la estructura anterior, obedecen a tres razones fundamentales:

- Responder con soluciones específicas a una situación crecientemente observada: el hecho de que la prestación se conceda, cada vez más como una ayuda orientada a complementar un bajo nivel de ingresos;

- Articular un sistema estable de prestaciones económicas complementarias que permita afrontar los gastos estructurales relacionados con la vivienda a las personas beneficiarias de rentas de garantía de ingresos, lo que a su vez, hace posible reorientar las ayudas de emergencia social hacia la cobertura de gastos extraordinarios;

- Implantar un modelo que, considerando el empleo como la mejor vía de inclusión, consiga hacer atractiva la incorporación al mercado laboral, incluso para acceder a un empleo de bajo nivel salarial.

Esta última razón, la centralidad del empleo como elemento de inclusión y la convicción de que, como tal, debe constituirse en elemento nuclear de los procesos orientados a la inclusión, es la que nos ha llevado a considerar la necesidad de reforzar su papel en el marco del sistema y de introducir modificaciones en el modelo de gestión que faciliten, realmente, en la práctica de los procesos, ese reforzamiento. De ahí que, aun asumiendo la Ley de 2008 como un avance en la dirección correcta, hemos optado por someterla a una reforma que traslada la gestión de las prestaciones de derecho y de los convenios de inclusión del ámbito de los servicios sociales al ámbito del empleo, recayendo en adelante dichas funciones en el servicio vasco de empleo-LANBIDE. Lógicamente, la apertura de esta vía quedaba condicionada a la previa transferencia de las competencias de las políticas activas de empleo desde la Administración del Estado al ejecutivo vasco, de ahí los esfuerzos, desde el inicio de la legislatura, por avanzar hacia ese objetivo.

El modelo que se propugna desde el Departamento se basa en la aplicación real del principio de doble derecho - a la percepción de una renta de garantía de ingresos y a la percepción de una serie de apoyos para la inclusión social y laboral- y en lo que algunos teóricos denominan principio de reciprocidad justa. Este principio reconoce que, para aplicar de forma legítima el criterio de condicionalidad que subyace a las prestaciones de garantía de ingresos, y que remite tanto a los derechos como a las responsabilidades de las personas perceptoras de esas prestaciones, las administraciones públicas deben garantizar un umbral mínimo de redistribución e igualdad de oportunidades, en especial en relación a la capacidad inclusiva del empleo y al funcionamiento del mercado de trabajo. En efecto, somos conscientes de que no se puede plantear el acceso al empleo como herramienta básica para la inclusión social y desatender al mismo tiempo las cuestiones relacionadas con la calidad del empleo y su capacidad inclusiva.

Somos conscientes de que la opción por este nuevo planteamiento genera, en unos y otros actores sociales, a la vez esperanzas y temores. Esperanzas, porque todo el mundo cree en la importancia del empleo como vector de inclusión, y porque, en mayor o menor medida, todos somos conscientes de que la inclusión laboral ha sido y es una asignatura pendiente en el sistema de rentas de garantía de

${ }^{6}$ Decreto $2 / 2010$, de 12 de enero, de la prestación complementaria de vivienda (BOPV de 19 de enero de 2010), Decreto 147/2010, de 25 de mayo, de la renta de garantía de ingresos (BOPV de 17 de junio de 2010), Decreto 4/2011, de 18 de enero, de las ayudas de emergencia social (BOPV de 9 de febrero de 2011). 
ingresos, a pesar de los innegables esfuerzos realizados a lo largo de todos estos años por los Servicios Sociales y a pesar de las experiencias de colaboración, coordinación e integración promovidas en algunas zonas del territorio autonómico. Temores, porque, más allá de la resistencia propia a los cambios, provoca dudas en las y los profesionales de los Servicios Sociales en relación con la capacidad de un servicio como LANBIDE, hasta ahora ajeno a las situaciones de grave exclusión, para responder, ajustadamente, a las muy particulares necesidades de estas últimas y para establecer cauces ágiles de coordinación y colaboración con los servicios sociales para atender estas situaciones; existe también, de forma bastante generalizada, un temor en relación con la aplicabilidad práctica de este modelo en un contexto económico en el que se observa un marcado y rápido aumento del desempleo y de la precariedad laboral.

En respuesta a estos temores diría que, desde nuestra óptica, esta nueva fórmula constituye la primera oportunidad real de situar la mejora de la empleabilidad en el centro de los procesos de inclusión, pero, sobre todo, insistiría en el hecho de que estas intervenciones dirigidas a la mejora de la empleabilidad en nada deben frenar los esfuerzos que desde los servicios sociales, los servicios sanitarios, los servicios educativos y los servicios de vivienda se orienten a la inclusión de quienes perciben la renta de garantía de ingresos. En realidad, lo que se pretende es que las políticas activas de empleo tengan vocación universal y atiendan a quienes acceden a la renta de garantía de ingresos, como también atienden al resto de la población, a imagen de lo que ocurre con las políticas públicas de salud, de vivienda, de educación o de Servicios Sociales. En cuanto a la capacidad del servicio vasco de empleoLANBIDE para dar cumplimiento a sus nuevas funciones, al margen del importante esfuerzo de formación y contratación, hemos desarrollado, a lo largo de 2011, un proyecto piloto de aplicación en un grupo de municipios para testar las posibilidades de asunción de la gestión de las prestaciones económicas y esa prueba ha confirmado nuestras expectativas en términos de capacidad de gestión. Por otra parte, estamos adoptando las medidas necesarias para que el tránsito de un sistema a otro sea fluido y se evite cualquier desajuste. Esto no significa, lógicamente, que el paso de un modelo a otro estará totalmente exento de dificultades, pero ¿qué modelo lo está?

\section{Conclusiones}

Son cambios de envergadura que, sinceramente y desde la responsabilidad, creo necesarios y beneficiosos tanto para las personas y familias que son usuarias del sistema vasco de Servicios Sociales y/o del sistema vasco de Garantía de ingresos y de inclusión social, como para el conjunto de la sociedad vasca. Requerirán, lógicamente, un tiempo de implantación y afianzamiento, pero apuntan en la buena dirección.

Creemos firmemente que avanzar por estas nuevas vías - ampliamente compartidas en Europa- nos dará nuevas claves de actuación, tanto en términos de innovación asistencial, en el marco de los Servicios Sociales, como en términos de inclusión laboral y mejora de la empleabilidad. Abrirán también nuevas oportunidades en el diseño de estrategias de coordinación, colaboración e integración de los esfuerzos con otras políticas públicas.

\section{Referencias bibliográficas}

Ley 12/2008, de 5 de diciembre, de Servicios Sociales (BOPV $n^{\circ} 246$, de 24 de diciembre de 2008).

Ley 18/2008, de 23 de diciembre, para la Garantía de Ingresos y para la Inclusión Social (BOPV $n^{\circ} 250$ de 31 de diciembre de 2008).

Decreto 64/2004, de 6 de abril, de Carta de Derechos y Obligaciones de las Personas Usuarias y Profesionales de los Servicios Sociales en la Comunidad Autónoma del País Vasco y el Régimen de Sugerencias y Quejas (BOPV $n^{\circ} 76$ de 23 de abril de 2004).

Decreto $2 / 2010$, de 12 de enero, de la prestación complementaria de vivienda (BOPV $n^{\circ} 11$ de 19 de enero de 2010).

Decreto 147/2010, de 25 de mayo, de la Renta de Garantía de Ingresos (BOPV $n^{\circ} 147$ de 17 de junio de 2010). 
Decreto 4/2011, de 18 de enero, de las Ayudas de Emergencia Social (BOPV $n^{\circ} 27$ de 9 de febrero de 2011).

Ararteko. (2010). La Situación de los Servicios Sociales de Base en la Comunidad Autónoma del País Vasco. Informe extraordinario de la institución del Ararteko al Parlamento Vasco. VitoriaGasteiz: Ararteko.

SIIS Centro de Documentación y Estudios de la Fundación Eguía-Careaga. (2011). Activación y derecho a la inclusión en el marco de las políticas de empleo y de garantía de ingresos en la CAPV. Documento de trabajo interno para el Departamento de Empleo y Asuntos Sociales del Gobierno Vasco. Donostia-San Sebastián: sine nomine.

\section{ANEXO I.}

Catálogo de Prestaciones y Servicios del Sistema Vasco de Servicios Sociales

1. Servicios sociales de atención primaria:

1.1. Servicio de información, valoración, diagnóstico y orientación.

1.2. Servicio de ayuda a domicilio.

1.3. Servicio de intervención socioeducativa y psicosocial. doras.

1.4. Servicio de apoyo a personas cuida-

1.5. Servicio de promoción de la participación y la inclusión social en el ámbito de los Servicios Sociales.

1.6. Servicio de teleasistencia.

1.7. Servicios de atención diurna.

1.8. Servicios de acogida nocturna.

1.9. Servicios de alojamiento:

1.9.1. Piso de acogida.

1.9.2. Vivienda tutelada.

1.9.3. Apartamentos tutelados.

1.9.4. Vivienda comunitaria.

\section{Servicios sociales de atención secun-} daria:

2.1. Servicio de valoración y diagnóstico de la dependencia, la discapacidad, la exclusión y la desprotección.

2.2. Servicios o centros de día.

2.2.1. Servicio o centro de día para atender necesidades derivadas de limitaciones en la autonomía.

2.2.2. Servicio o centro ocupacional.

2.2.3. Servicio o centro de día para atender necesidades de inclusión social.

2.3. Centros de acogida nocturna.

2.3.1. Centro de noche para atender necesidades derivadas de limitaciones en la autonomía.

2.3.2. Centro de acogida nocturna para atender necesidades de inclusión social.
2.4. Centros residenciales.

2.4.1. Centros residenciales para personas mayores.

2.4.2. Centros residenciales para personas con discapacidad.

2.4.3. Centros residenciales para personas con enfermedad mental.

2.4.4. Centros residenciales para personas menores de edad en situación de desprotección.

2.4.5. Centros residenciales para personas en situación de exclusión y marginación.

2.4.6. Centros residenciales para mujeres víctimas de maltrato doméstico y otros servicios residenciales para mujeres.

2.5. Servicio de respiro.

2.6. Servicio de coordinación a urgencias sociales.

2.7. Otros servicios de atención secundaria.

2.7.1. Servicios de información y orientación.

2.7.1.1. Servicio de información social a la infancia y la adolescencia en situación de desprotección.

2.7.1.2. Servicio de información y atención a mujeres víctimas de violencia doméstica o por razón de sexo.

2.7.2. Servicios de soporte de la autonomía.

2.7.2.1. Servicio de apoyo a la vida independiente.

2.7.2.2. Servicio de ayudas técnicas y adaptación del medio físico.

2.7.2.3. Servicio de tutela para personas adultas incapacitadas.

2.7.2.4. Servicio de transporte adaptado.

2.7.3. Servicios de intervención y mediación familiar. 
2.7.3.1. Servicios de intervención socioeducativa y/o psicosocial con familias.

2.7.3.2. Punto de encuentro familiar.

2.7.3.3. Servicio integral de mediación familiar.

2.7.4. Servicio de intervención social en atención temprana.

2.7.5. Servicios de atención sociojurídica y psicosocial de las situaciones de maltrato doméstico y agresiones sexuales a mujeres, a personas menores de edad, a personas mayores y a personas con discapacidad.

2.7.6. Servicios de promoción y apoyo al acogimiento familiar y la adopción.

2.7.6.1. Servicio de promoción y apoyo técnico al acogimiento familiar.

2.7.6.2. Servicio de promoción y apoyo técnico a la adopción.

\section{Prestaciones económicas:}

3.1. Prestaciones para facilitar la integración social y/o la autonomía así como para cubrir o paliar situaciones de emergencia social.

3.2. Prestaciones para apoyar y compensar a las personas que ofrecen apoyo social informal.

3.3. Prestaciones para la adquisición de prestaciones tecnológicas:

3.4. Prestaciones vinculadas a servicios personales.

3.5. Otras prestaciones económicas que puedan establecerse en el marco de las finalidades propias del Sistema Vasco de Servicios Sociales.

\section{Plan Estratégico de Servicios Sociales: ejes básicos y objetivos estratégicos}

\section{Eje 1. Universalización de la atención, or- denación territorial y equidad en el acceso}

Objetivos:

- Avanzar en la adecuación de las coberturas de atención de los servicios y prestaciones del catálogo de Servicios Sociales a las demandas de atención detectadas, así como en la ordenación territorial de los servicios.

- Adecuar los ratios de atención y/o la intensidad de los diversos servicios para su adecuación a la normativa vigente.

- Aplicar o, en su caso, desarrollar los instrumentos comunes de acceso a las diversas prestaciones.

- Avanzar en la mejora de la accesibilidad de los centros de Servicios Sociales.

- Implementar planes de prevención que eviten o retarden la necesidad del acceso a determinados ámbitos de los Servicios Sociales, así como estudios periódicos de previsión que posibiliten una política de servicios sociales ajustada a las necesidades sociales y a los recursos disponibles.

- Incorporar la perspectiva de género a todas las acciones que forman parte del Plan, al objeto de eliminar las desigualdades de género y promover la igualdad de mujeres y hombres en el ámbito de los Servicios Sociales.
Eje 2. Afianzamiento y desarrollo del enfoque comunitario y del procedimiento básico de intervención

Objetivos:

- Mejorar la capacidad del sistema para ofrecer a las personas usuarias de los Servicios Sociales que lo precisen un acompañamiento social personalizado e integral desde los Servicios Sociales de Base.

- Mejorar el apoyo prestado a las personas cuidadoras.

- Facilitar la permanencia de las personas con discapacidad o dependencia en su domicilio mediante el desarrollo de las nuevas tecnologías de apoyo y la provisión de ayudas técnicas.

- Reforzar las labores de intervención y prevención comunitaria desde los Servicios Sociales.

- Avanzar en la adecuación de las características, el diseño y la ubicación de los equipamientos, en especial los de carácter residencial, al enfoque comunitario y al principio de proximidad, de acompañamiento social personalizado e integral, y de atención centrada en la persona.

- Potenciar la prevención de las situaciones de desprotección infantil mediante programas de preservación e intervención familiar. 
- Promover la inclusión social y la autonomía de las personas usuarias y potenciar la participación social enfocada desde la solidaridad.

Eje 3. Eficiencia: modernización y optimización de los recursos

Objetivos:

- Reducir la complejidad del sistema, especialmente desde el punto de vista de las personas usuarias, desarrollando fórmulas de gestión administrativa que permitan una tramitación más ágil de las demandas.

- Promover la mejora de la calidad y la eficiencia de los servicios mediante las actividades de I+D y la potenciación de las buenas prácticas en la gestión y la prestación de servicios.

- Favorecer un aprovechamiento de los recursos humanos, mejorando la productividad y posibilitando una mayor calidad de la atención.

- Promover unas condiciones laborales adecuadas, en un entorno sostenible, a todas las personas que intervienen en la prestación de Servicios Sociales de responsabilidad pública.

\section{Eje 4. Articulación interna del sistema y mejora de la calidad}

Objetivos:

- Afianzar las estructuras internas necesarias para el desarrollo del Sistema Vasco de Servicios Sociales.

- Fomentar las actividades dirigidas a la mejora de la calidad en la prestación de los Servicios Sociales.

- Mejora continua de la formación y cualificación de las personas que trabajan en el ámbito de los servicios sociales, así como del voluntariado y de las personas cuidadoras.

\section{Eje 5. Conocimiento por parte de la ciuda-} danía y participación social

Objetivos:

- Mejorar el grado de conocimiento del sistema de Servicios Sociales por parte de la población y favorecer una percepción de los
Servicios Sociales como sistema coordinado y unificado.

- Extender los canales para la participación ciudadana $\mathrm{y}$, en especial, de las personas usuarias en el desarrollo de las políticas de Servicios Sociales y alcanzar mayores cotas de protagonismo de las personas usuarias no sólo en las Políticas de Servicios Sociales sino en la concepción y funcionamiento de los recursos.

- Impulsar la participación del voluntariado en la definición y la gestión de los servicios sociales, y reconocer su aportación.

Eje 6. Cooperación con los servicios de salud, educación, vivienda, justicia y empleo

Objetivos:

- Culminar la construcción del espacio sociosanitario, dando la representación y cobertura necesaria a todos los colectivos tributarios de su atención, y mejorar la coordinación entre los servicios sociales y los de salud.

- Mejorar el trabajo en red con los servicios de empleo.

- Mejorar el trabajo en red con los centros escolares y establecer acuerdos de colaboración conjunta.

- Mejorar la coordinación con otros sistemas (justicia, vivienda, transporte...), dada la transversalidad de las necesidades de los colectivos atendidos por los servicios sociales.

\section{Eje 7. Colaboración entre el sector público} y el privado

Objetivos:

- Definir el marco regulador para la prestación de servicios de responsabilidad pública por parte de las entidades privadas con y sin fin de lucro.

- Impulsar el desarrollo de la responsabilidad social corporativa en el ámbito de los Servicios Sociales en la CAPV.

- Promover la inclusión social y la autonomía de las personas usuarias y potenciar la participación social enfocada desde la solidaridad. 
ANEXO III.

Mapa de Servicios Sociales: grados de proximidad de los servicios y prestaciones

\begin{tabular}{|c|c|c|}
\hline Grado de proximidad & Ámbito territorial & Servicios de la Cartera \\
\hline $\begin{array}{c}\text { Servicios } \\
\text { centralizados }\end{array}$ & $\begin{array}{l}\text { Comunidad } \\
\text { Autónoma }\end{array}$ & $\begin{array}{l}\text { 2.7.1.1. Servicio de información social a la infancia y a la adolescencia } \\
\text { en situación de desprotección } \\
\text { 2.7.1.2. Servicio de información y atención a mujeres víctimas de la vio- } \\
\text { lencia doméstica o por razón de sexo }\end{array}$ \\
\hline $\begin{array}{l}\text { Servicios } \\
\text { de baja } \\
\text { proximidad }\end{array}$ & $\begin{array}{l}\text { Territorio } \\
\text { Histórico }\end{array}$ & $\begin{array}{l}\text { 1.6. Servicio de teleasistencia } \\
\text { 1.8. Servicios de acogida nocturna } \\
\text { 2.1. Servicio de valoración y diagnóstico de la dependencia, la discapaci- } \\
\text { dad, la exclusión y la desprotección } \\
\text { 2.2.3. Servicio o centro de día para atender necesidades de inclusión social } \\
\text { 2.3.2. Centro de acogida nocturna para atender necesidades de inclusión } \\
\text { social } \\
\text { 2.4.5. Centros residenciales para personas en situación de exclusión y } \\
\text { marginación } \\
\text { 2.4.6. Centros residenciales para mujeres } \\
\text { 2.6. Servicios de coordinación de urgencias sociales } \\
\text { 2.7.2.1. Servicio de apoyo a la vida independiente } \\
\text { 2.7.2.2. Servicio de ayudas técnicas y adaptación del medio físico } \\
\text { 2.7.2.3. Servicio de tutela para personas adultas incapacitadas } \\
\text { 2.7.2.4. Servicio de transporte adaptado } \\
\text { 2.7.3.3. Servicio de mediación familiar } \\
\text { 2.7.6.1. Servicio de promoción y apoyo técnico al acogimiento familiar } \\
\text { 2.7.6.2. Servicio de promoción y apoyo técnico a la adopción } \\
\text { 3.1. Prestaciones para facilitar la integración social y/o autonomía (...) } \\
\text { 3.2. Prestaciones para apoyar y/o compensar a las personas que ofrecen } \\
\text { apoyo social informal } \\
\text { 3.4. Prestaciones vinculadas a servicios personales }\end{array}$ \\
\hline $\begin{array}{l}\text { Servicios } \\
\text { de proximidad } \\
\text { media }\end{array}$ & $\begin{array}{c}\text { Sector } \\
\text { de Servicios } \\
\text { Sociales } \\
\text { Entre } 200.000 \\
\text { y } 400.000 \\
\text { habitantes }\end{array}$ & $\begin{array}{l}\text { 1.7. Servicios de atención diurna } \\
\text { 2.3.1. Centro de noche para atender necesidades derivadas de limitacio- } \\
\text { nes en la autonomía } \\
\text { 2.4.5. Centros residenciales para personas en situación de exclusión y } \\
\text { marginación } \\
\text { 2.4.6. Centros residenciales para mujeres } \\
\text { 2.7.3.2. Punto de encuentro familiar } \\
\text { 2.7.4. Servicio de intervención social en atención temprana }\end{array}$ \\
\hline $\begin{array}{l}\text { Servicios } \\
\text { de alta } \\
\text { proximidad }\end{array}$ & $\begin{array}{c}\text { Área } \\
\text { de Servicios } \\
\text { Sociales } \\
\text { Regla general: } \\
\text { Entre } 15.000 \\
\text { y } 50.000 \\
\text { habitantes } \\
\text { (con excepciones } \\
\text { aplicables } \\
\text { a municipios } \\
\text { grandes) }\end{array}$ & $\begin{array}{l}\text { 1.9.1. Pisos de acogida } \\
\text { 1.9.2. Vivienda tutelada } \\
\text { 1.9.3. Apartamentos tutelados } \\
\text { 1.9.4. Vivienda comunitaria } \\
\text { 2.2.1. Servicio o centro de día para atender necesidades derivadas de li- } \\
\text { mitaciones en la autonomía } \\
\text { 2.2.2. Servicio o centro ocupacional } \\
\text { 2.4.1. Centros residenciales para personas mayores } \\
\text { 2.4.2. Centros residenciales para personas con discapacidad } \\
\text { 2.4.3. Centros residenciales para personas con enfermedad mental } \\
\text { 2.4.4. Centros residenciales para personas menores de edad en situación } \\
\text { de desprotección } \\
\text { 2.5. Servicios de respiro } \\
\text { 2.7.3.1. Servicios de intervención socioeducativa y psicosocial con familias } \\
\text { 2.7.5. Servicios de atención sociojurídica y psicosocial a las situaciones } \\
\text { de maltrato doméstico y agresiones sexuales a mujeres, personas meno- } \\
\text { res de edad, a personas mayores y a personas con discapacidad }\end{array}$ \\
\hline
\end{tabular}




\begin{tabular}{ccl}
\hline Grado de proximidad & Ámbito territorial & \multicolumn{1}{c}{ Servicios de la Cartera } \\
\hline Servicios & Zona Básica de & 1.1. Servicio de información, valoración, diagnóstico y orientación \\
de máxima & Servicios Sociales: & 1.2. Servicio de ayuda a domicilio \\
proximidad & Urbanas: entre & 1.3. Servicio de intervención socioeducativa y psicosocial \\
& 5.000 y 15.000 & 1.4. Servicio de apoyo a personas cuidadoras \\
& habitantes & 1.7. Servicios de atención diurna \\
& Rurales: entre & 2.1. Servicio o centro de día para atender necesidades derivadas de li- \\
& 3.000 y 7.000 & 2.2.1. \\
& habitantes & mitaciones en la autonomía \\
\hline
\end{tabular}

